\title{
MedChemComm
}

CORRECTION
View Article Online

View Journal | View Issue

\section{Correction: Analysis of the quantitative structure- activity relationship of glutathione-derived peptides based on different free radical scavenging systems}

Cite this: Med. Chem. Commun., 2016, 7, 2193

\author{
Wenzhen Liao, ${ }^{a}$ Longjian Gu, ${ }^{a}$ Yamei Zheng, ${ }^{a}$ Zisheng Zhu, ${ }^{a}$ Mouming Zhao, ${ }^{a}$ \\ Ming Liang ${ }^{b}$ and Jiaoyan Ren*a
}

DOI: $10.1039 / \mathrm{c} 6 \mathrm{md} 90050 \mathrm{j}$

Correction for 'Analysis of the quantitative structure-activity relationship of glutathione-derived peptides based on different free radical scavenging systems' by Wenzhen Liao et al., Med. Chem. Commun., 2016,

www.rsc.org/medchemcomm DOI: 10.1039/c6md00006a.

The authors regret that some corrections had not been made to the final manuscript. Please find the required corrections listed below:

In section 2.7 Statistical analysis, the final sentence 'The MAE based criteria......the QSAR model. ${ }^{24}$, should be replaced by: 'The MAE based criteria were used for the training set using cross-validated leave-one-out (LOO) predictions for additional validation of the QSAR models. ${ }^{24,58}$ The software tool to perform the MAE based test was downloaded from the website: http:// teqip.jdvu.ac.in/QSAR_Tools/.'

The above revised sentence includes a new reference: 58. K. Roy, R. N. Das, P. Ambure and R. B. Aher, Chemom. Intell. Lab. Syst., 2016, 152, 18-33.

In section 3.5.1 Predictive capability of the models, the sentence 'Therefore, external validation.......high predictive power.' should be replaced by: 'However, in the absence of sufficient number of data points for external validation, the MAE based criteria were applied for cross-validation, and the results proved that the QSAR model for ORAC database exhibited a high predictive power.'

In Table 4 the title of the final column 'External validation test (MAE)' should be replaced by: 'MAE based criteria'.

The Royal Society of Chemistry apologises for these errors and any consequent inconvenience to authors and readers.

\footnotetext{
${ }^{a}$ Department of Food Science and Technology, College of Food Sciences and Technology, South China University of Technology, Wushan Road 381, Guangzhou 510640, Guangdong, China. E-mail: jyren@scut.edu.cn; Fax: +86 20 38897117; Tel: +86 2087112594

${ }^{b}$ R\&D Center, Infinitus (China) Co., LTD., Guangzhou 510665, China
} 